\title{
Object Shape Recognition from EEG Signals during Tactile and Visual Exploration
}

\author{
Anwesha Khasnobish ${ }^{1}$, Amit Konar ${ }^{2}$, D.N. Tibarewala ${ }^{1}$, \\ Saugat Bhattacharyya ${ }^{2}$, and R. Janarthanan ${ }^{3}$ \\ 1 School of Bioscience and Engineering, Jadavpur University, Kolkata \\ ${ }^{2}$ Department of Electronics and Telecommunication Engineering, \\ Jadavpur University, Kolkata \\ 3 Department of Computer Science \\ TJS Engineering College, Chennai, India \\ \{anweshakhasno, biomed.ju, saugatbhattacharyya@gmail.com\}, \\ konaramit@yahoo.co.in, srmjana_73@yahoo.com
}

\begin{abstract}
Humans understand the world around us by visual and tactile exploration of the objects. The objective of this paper is to recognize the object-shapes from EEG signals while the subjects are exploring the same by visual and tactile means. The various object shapes are classified from electroencephalogram (EEG) signals that are stimulated by only tactile, only visual and by both means. EEG signals were acquired and analyzed from six electrodes, namely F3,F4,FC5,FC6,O1 and O2, where each pair of electrodes are located on frontal, somato-sensory and occipital region of the brain responsible for cognitive processing, tactile and visual perception. Mu-desynchronization in alpha and beta bands is used as the EEG modality for this purpose. Power spectral density (PSD) features are extracted and classified using support vector machine (SVM) classifiers in their corresponding object-shape classes. The results showed that object-shapes are best classified from EEG signals during only tactile exploration. The object shapes classified from EEG signals during only tactile exploration yielded highest mean classification accuracy of $88.34 \%$. The average classification accuracy over all three object exploration modalities is $83.89 \%$.
\end{abstract}

Keywords: Tactile perception, visual perception, object-shape recognition, electroencephalogram, power spectral density, support vector machine.

\section{Introduction}

Humans perceive their real world both by visual examination and manual exploration of the objects around them [1], [2], [3], [4]. This paper is targeted to recognize object shapes directly from EEG signals during visual and tactile exploration of the same objects. This study can find its applications in various areas of human computer interactions, tele-operation, and virtual reality. This study will also be helpful for blind (for whom visual examination is absent) as 
well as autistic patients (who are unable to express the information about the object perceived).

Electroencephalogram (EEG) provides an interesting means to understand brain functionality during visual and haptic percep-tion [5], [6], [7]. This paper considers EEG signal acquisition during only tactile exploration, only visual exploration and their joint consideration to recognize objects from EEG signals. The mu-desynchronization in alpha and beta band is out to be of im-portance of FFT analysis of the corresponding EEG signals during visual and haptic object exploration. EEG electrodes located particularly at frontal, somato-sensory, and occipital regions of the human brain following the traditional 10-20 EEG electrode placement system have been used for the present work. The electrode positions F3 and F4 refer to cognitive basis while FC5 and FC6 correspond to tactile and $\mathrm{O} 1$ and $\mathrm{O} 2$ correspond to visual basis of perception. Support vector machine (SVM) classifiers have been employed to recognize object-shapes from only tactile, only visual and the composite extracted power spectral density features. Experimental results indicate that only tactile perception provides the better basis of recognizing object shapes than only visual and composite modality.

The paper is divided in five sections. The selection of EEG modality and frequency bands and electrode localization is pre-sented in section 2 . The undertaken methodology of feature extraction and classification of object shapes is explained in section 3 . The section 4 depicts the experimental paradigm along with the results. Concluding remarks are included in section 5 .

\section{EEG Modalities for Visual/Tactile Perception}

Performing FFT analysis on our data set, we chose the desynchronization of the mu rhythms for analysis of the tactile and visually stimulated event related potentials of EEG signals. It is observed that there occurs a decline in the EEG rhythms in mu-band on the onset of visual or tactile stimuli presented to the subjects. This is referred to as mu-ERD. Thus, we have considered these desynchronizations of mu signals as the EEG modality for analyzing tactile and visually stimulated events

In this work we are concerned with both visual and tactile perception for recognizing shapes. The visual perception originates from the occipital lobe; the tactile i.e. somatosensory perception is related to the somatosensory cortex. Along with these, cognitive processing and understanding is associated with frontal lobe of human brain. EEG signals from these areas are acquired from F3, F4, FC5, FC6, O1 and O2 electrodes which lie on the scalp over the frontal, somatosensory and occipital lobes respectively [8]. Since the dominant frequency bands for mu rhythm is mainly $8-13 \mathrm{~Hz}$ and also $15-25 \mathrm{~Hz}$, we have thus considered EEG signals in alpha $(8-13 \mathrm{~Hz})$ and central beta band $(15-25 \mathrm{~Hz})$ for further analysis. 


\section{EEG Feature Extraction and Classification}

For the EEG signals within frequency range $8-25 \mathrm{~Hz}$, power spectral density (PSD) (9) features are extract-ed for classification. The Welch approach was applied along with a Hamming window of length 64 . The Welch method divides the times series data into overlapping segments, computing a modified periodogram of each segment and then the PSD estimates is averaged. Also the average power was obtained for each band.

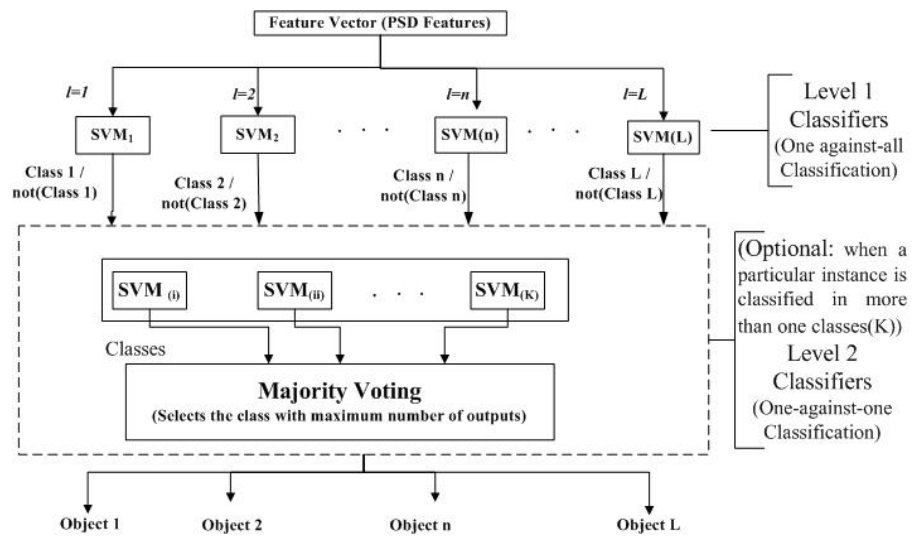

Fig. 1. One-against-all classification approach using linear SVM

The extracted PSD features from the EEG data were classified using support vector machine (SVM) [10], [11] into their respective object-shape classes. Using SVM we have classified the extracted PSD feature vectors in corresponding object classes (Fig.1). This is done for all the three experimental phases (i.e. only tactile exploration, only visual examination and by both means), which is explained in the section IV. Number of object classes considered in this study is $l$, (where $l=1$ toL, $L=10$ ). For example, the instance of object 3(say) will be classified by SVM3 classifier, with output as Class 3 and the rest of the SVM classifiers will produce the output as not(class 3 ). The classification is performed in two stages. In the first level, the extracted PSD features are classified using L number SVM classifiers on one-against-all basis in their corresponding classes (Fig 2). Each SVM classifier, $S V M_{n}$, classified the feature vector in class $\mathrm{n}$ or not-n, where $n=1$ toL i.e., according to the number of object classes. In ideal cases a particular instance will be classified in only one class (among all $l, l=1 t o L)$. If a particular instance after classification by Level 1 classifiers, is classified in more than one classes then the Level 2 classification is performed. For example, if a particular instant is classified in class 1, 3, 5 and 8 . When next level of classification is performed to determine the actual class in which that particular instance belongs. In Level $2, K=n_{r}^{C}$ number of SVM classifiers are employed, where $n$ is the number of classes in which the particular instance is 
classified (in the above example $n=4$ ) and $r=2$ since in this stage SVM will classify in one-against-one approach. The output clas-ses of these Level 2 SVM classifiers are fed in a majority voting system, which selects the class with maximum number of occurrences, which is the actual class in which that particular instance belongs. If there occurs no such class with more number of oc-currences than the other classes, then that particular instance is rejected. For this classifier system the cost $(c)=1.00$ and gamma $=0.00$ is set.

\section{Experiments and Results}

The experimental framework and the results obtained from them are illustrated in this section.

Framework. EEG signals are recorded from 25 subjects (17 male and 8 female) of age group 243 years. We considered 10 objects of different shapes as shown in Fig. 3. Our object set consisted of regular geometrical shapes such as cone, cube, sphere, hemisphere, cylinder, prism, and hexagonal base cylinder. Two irregular shaped objects i.e. a lock and a mouse is also included.EEG was recorded using a 14 channel Emotive EEG Research edition SDK with sampling rate of $128 \mathrm{~Hz}$. Out of the 14 channels we have considered F3, F4, FC5, FC6, O1 and O2 for our analysis purpose.

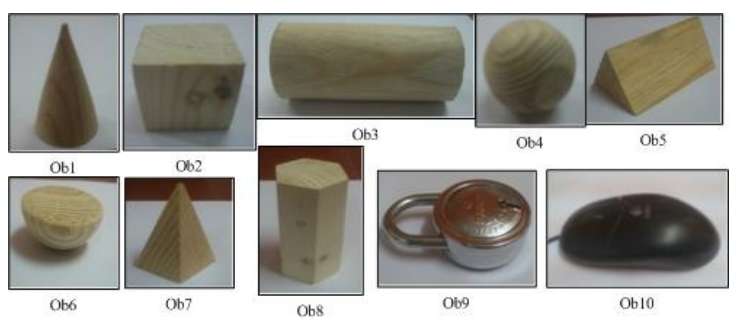

Fig. 2. Objects considered for experiments: Ob1. Cone, Ob2. Cube, Ob3. Cylinder, Ob4. Sphere, Ob5. Prism, Ob6. Hemisphere, Ob7. Pyramid (square base), Ob8. Hexagonal base cylinder, Ob9. Lock, and Ob19. Mouse.

The EEG data were recorded during three experimental phases consisting of only tactile exploration, followed by only visual examination and exploring the object by both visual and tactile means. Audio-Visual cues were presented to direct the subject to explore the object in the sequential manner for 10 seconds duration. A blank screen of 30seconds duration is followed by a fixation cross, directing the subject to focus on the screen. During only tactile exploration phase, the fixation cross is followed by an instruction to close the subjects eye, then the subject was given an object in his palm to dynamically explore by touching for 10seconds. A beep sound at the end of ten seconds prompts the 
user to stop exploration and open his/her eyes. Then again a blank screen for 5 seconds is followed by the fixation cross and the sequence is repeated for exploring all the 10 objects. During visual examination, the fixation cross is followed by an image of the object on the screen for 10 seconds. During object exploration by both tactile and visually, the subject is prompted to dynamically explore the object by touching at the same time he/she can visually examine the same. During each phase, each object is examined 10 times for 10 seconds. Thus during each exploration phase (i.e. either visual, or tactile or both), for each subject data were recorded for 100 trials (10 objects examined 10 times) of 10 seconds each. The sampling rate was $128 \mathrm{~Hz}$, thus we have 6400 data points for each object.

Performance Analysis. 10- fold cross validation is performed to divide the PSD features (see section 3) into training and testing instances and fed in the SVM classifiers.

Table 1. Classification Accuracies (in \%)

\begin{tabular}{cccc}
\hline Objects (a) & Only tactile (b) & Only visual (c) & Both tactile and visual \\
\hline Ob1 & 77.8 & 77.8 & 88.9 \\
Ob2 & 77.8 & 88.9 & 88.9 \\
Ob3 & 88.9 & 77.8 & 77.8 \\
Ob4 & 88.9 & 66.7 & 66.7 \\
Ob5 & 100.0 & 87.5 & 75.0 \\
Ob6 & 87.5 & 87.5 & 87.5 \\
Ob7 & 100.0 & 75.0 & 87.5 \\
Ob8 & 87.5 & 87.5 & 87.5 \\
Ob9 & 100.0 & 75.0 & 87.5 \\
Ob10 & 75.0 & 87.5 & 75.0 \\
\hline Mean & 88.3 & 81.1 & 82.2 \\
\hline
\end{tabular}

The results depicted in Table.1, shoe that the only tactile exploration yielded highest mean accuracy of $88.34 \%$. The average classification accuracy over all three object exploration modalities is $83.89 \%$.

\section{Conclusion}

In this paper we presented a novel approach to recognize object shapes from EEG signals during only visual, only tactile and both tactile-visual exploration. The extracted PSD features are classified using two-level SVM classifiers in corresponding object-shape classes for the three experimental phases. 
The results reveal that only tactile exploration provides subjects with every detail of the object, thus object shape recognition rate is the highest. It is also evident from the results that any of the three object-exploration modalities is sufficient to classify objects shapes from EEG signals. The visual perception does not carry information of the object shape for the hidden surfaces and edges, which contributes in lowest recognition rate while examining the objects only visually. Though in general we experience object recognition seems to be faster by only visual examination, but that is achieved through years of learning process in our brain. In case on exploring the objects by both visual and tactile means number of information increases to a large amount due to combination of both visual and tactual information, which results in increased misclassifications. Further study in this field will be performed considering more number of subjects and objects with new tools and techniques.

Acknowledgments. This study has been supported by University Grants Commission, India, University with Potential for Excellence Program (UGC-UPE) (Phase II) in Cognitive Science, Jadavpur University and Council of Scientific and Industrial Research (CSIR), India.

\section{References}

1. Cristino, F., Colan, L.I., Leek, C.E.: The appearance of shape in visula perception: eye movement patterns during recognition and reaching. In: 3rd International Conference on Appearance, UK, pp. 125-127 (2012)

2. Hoosiang, H.: Differential surface models for tactile perception of shape and online tracking of features. IEEE Trans System, Man and Cybernetics 18(2), 312-316 (1988)

3. Szubielsca, M.: Prior visual experience, and perception and memory of shape in people with total blindness. British J. Visual Impairment 29, 60-81 (2011)

4. Santhian, K.: Visual cortical activity during during tactile perception in sighted and visually deprived. J. Developmental Psychobiology 46(3), 279-286 (2005)

5. Mustafa, M., Lindemann, L., Magnor, M.: EEG Analysis of Implicit Human Visual Perception. In: ACM Human Factors in Computing Systems, CHI (2012)

6. Christopher, P., Taylor, J., Thut, G.: Brain activity underlying visual perception and attention as inferred from TMS-EEG: A review. J. Brain Stimulation 5(2), $125-129$ (2012)

7. Grunwald, M., et al.: Theta power in the EEG of humans during ongoing processing in a haptic object recognition task. J. Cog. Brain Research 11, 33-37 (2001)

8. Teplan, M.: Fundamentals of EEG Measurement. J. Measurement Sc. Review 2(2) (2002)

9. Herman, P., Prasad, G., McGinnity, T.M., Coyle, D.: Comparative analysis of spectral approaches to feature extraction for EEG-based motor imagery classification. IEEE Trans. Neural Sys. Rehab Eng. 16(4), 317-326 (2008)

10. Gunn Steve, R.: Support Vector Machines For Classification and Regression. Technical report, University of Southampton (1998)

11. Burges Christopher, J.C.: A Tutorial on Support Vector Machines for Pattern Recognition. Kluwer Academic Publishers, Boston 